\title{
Effects of high-dose large neutral amino acid supplementation on exercise, motor skill, and mental performance in Australian Rules Football players
}

\author{
Nigel K. Stepto, Benjamin B. Shipperd, Graeme Hyman, Bernie Mclnerney, and \\ David B. Pyne
}

\begin{abstract}
This study investigated the effects of high-dose large neutral amino acid (LNAA) supplementation on attenuating fatigue-induced decrements in exercise and motor skill performance in Australian Rules Football (ARF) players. Fifteen subelite ARF players participated in 3 testing sessions separated by 7 days. Players completed an initial control trial involving a reactive motor skills test (RMST) and a reactive agility test (RAT) carried out before and after fatiguing exercise. In the subsequent experimental trials, players ingested a serotonin-depleting or protein control (PC) LNAA mixture $3 \mathrm{~h}$ before testing, allocated in a double-blind randomized cross-over design. Blood samples were taken at presupplementation and pre- and postexercise for analysis of plasma amino acid, insulin, and metabolite concentrations. The effect of the LNAA was established as the difference in the change in the mean RMST and RAT test scores among the depleting, PC, and baseline (BL) trials. Mean overall repetition time of the RAT was moderately improved by $-5.2 \% \pm 3.4 \%$ (mean $\pm 90 \%$ confidence limits; effect size $-0.45 \pm 0.28$ ) after ingestion of the serotonin-depleting mixture compared with the BL trial. Serotonin-depleting and PC supplements had a divergent effect on mean repetition time after fatiguing exercise in RMST: depleting serotonin elicited a small improvement $(-3.0 \% \pm 2.7 \%)$ in motor skill performance in contrast to a small decrement $(2.4 \% \pm 2.7 \%)$ after ingestion of the PC mixture, when compared to the BL. High-dose serotonin-"depleting" LNAA supplementation given $3 \mathrm{~h}$ prior to intermittent high-intensity exercise improved reactive motor skill and agility performance in ARF players.
\end{abstract}

Key words: team sport, fatigue, serotonin, tryptophan, cognitive function, mood states.

Résumé : Cette étude analyse les effets de fortes doses d'un supplément constitué de grands acides aminés neutres (LNAA) sur la baisse de fatigue à l'effort et sur la performance motrice chez des joueurs de football australien (ARF). Quinze joueurs de niveau sous-élite participent à trois séances d'évaluation à sept jours d'intervalle chacune. À la première séance (contrôle), les joueurs passent des tests d'habiletés motrices réactives (RMST) et d'agilité réactive (RAT), et ce, avant et après un exercice épuisant. Au cours des séances subséquentes, les sujets consomment $3 \mathrm{~h}$ avant les tests un mélange de LNAA affaissant la sérotonine ou un mélange de contrôle constitué de protéines (PC); les suppléments sont administrés aléatoirement selon un plan croisé à double insu. Avant la supplémentation ainsi qu'avant et après l'exercice, on prélève des échantillons de sang pour analyser le contenu plasmatique en acides aminés, en insuline et en métabolites. On évalue l'effet de LNAA d'après les variations des résultats moyens au RMST et au RAT aux séances dites d'affaissement, de PC et de référence (BL). Le temps global moyen de répétition au RAT s'améliore modérément de $-5,2 \pm 3,4 \%$ (moyenne \pm limites de confiance à $90 \%$; ampleur de l'effet $-0,45 \pm 0,28$ ) après la consommation du mélange affaissant la sérotonine, comparativement à la séance BL. Les suppléments affaissant la sérotonine et de PC n'ont pas le même effet sur le temps de répétition au RMST suivant l'exercice épuisant : le mélange affaissant la sérotonine suscite une faible amélioration $(-3,0 \pm 2,7 \%)$ de la performance motrice alors que le mélange de PC suscite une faible détérioration $(2,4 \pm 2,7 \%)$ de la performance motrice, comparativement aux valeurs de BL. Une forte dose d'un supplément de LNAA affaissant la sérotonine et administré $3 \mathrm{~h}$ avant un exercice intermittent de haute intensité améliore la réactivité des habiletés motrices et de l'agilité chez des joueurs de football australien (ARF).

Mots-clés : sport d'équipe, fatigue, sérotonine, tryptophane, fonction cognitive, état d'esprit.

[Traduit par la Rédaction]

Received 10 March 2011. Accepted 3 June 2011. Published at www.nrcresearchpress.com/apnm on 7 October 2011.

N.K. Stepto. Institute of Sport Exercise and Active Living and School of Sport and Exercise Science, Victoria University, P.O. Box 14428, Melbourne, Victoria, 8001 Australia; School of Sport and Exercise Science, Victoria University, Melbourne, Victoria, Australia.

B.B. Shipperd. School of Physiotherapy, Monash University, Frankston, Victoria, Australia.

G. Hyman. Department of Psychology, Monash University, Caulfield, Victoria, Australia.

B. McInerney. Australian Proteome Analysis Facility, Macquarie University, Sydney, New South Wales, Australia.

D.B. Pyne. Department of Physiology, Australian Institute of Sport, Belconnen, Australian Captial Territory, Australia.

Corresponding author: Nigel Stepto (e-mail: Nigel.Stepto@vu.edu.au). 


\section{Introduction}

Fatigue in team sports typically accumulates over the course of a game, affecting the amount and intensity of exercise performed, skill execution, and decision making. Other studies of team sports, especially football (soccer), have demonstrated that the volume of high- and very high-intensity running is reduced as the game progresses (Mohr et al. 2003; Rampinini et al. 2007; Spencer et al. 2004). High-intensity exercise (including game time) is associated with declines in passing skill performance in elite and junior soccer players (Rampinini et al. 2008, 2009). Nutritional intervention(s) prior to and during the course of a game that attenuate the negative effects of fatigue are a practical and effective way of maintaining high levels of performance.

The causes of fatigue are complex and often involve a combination of both central and peripheral factors. Peripheral fatigue relates to impairments of calcium release from the sarcoplasmic reticulum, ionic homeostasis and membrane excitability, mechanisms of cross-bridge cycling, and metabolic and contractile processes in skeletal muscle (Allen et al. 2008; Burke and Hawley 1999; Welsh et al. 2002). Inadequate availability of fuels (whether from endogenous or exogenous sources) also influences peripheral fatigue (Burke and Hawley 1999). However, fatigue can occur in the absence of any peripheral influences (Davis and Bailey 1997), implicating a centrally based or central nervous system (CNS) mechanism. Factors leading to CNS-based fatigue include any processes within the CNS that have downstream effects on muscle function, such as impaired neural propagation of stimuli from the brain and spinal cord (Gandevia 2001). The mechanisms involved in CNS-based fatigue, and the extent to which these affect exercise performance, are not clear.

The central fatigue hypothesis implicates altered levels of serotonin in the brain as the cause of impaired performance (Blomstrand et al. 1988, 1989; Young et al. 1985). Serotonin is considered a key neurotransmitter causing central fatigue because pharmacological enhancement of its neurotransmission can exacerbate exercise-induced fatigue (Weicker and Struder 2001). Conversely, lowering brain serotonin levels delays the onset of exercise-induced fatigue (Yamamoto and Newsholme 2000). Given that serotonin is produced from tryptophan, nutritional strategies can be used to manipulate brain serotonin levels. This effect is achieved by ingesting a mixture of large neutral amino acids (LNAA), including the branched-chain amino acids (BCAA) (Nathan et al. 2004; Nishizawa et al. 1997). Tryptophan, and therefore serotonin, depletion is achieved by competitively inhibiting the LNAA transporters with a high dose of BCAA not containing tryptophan (Pardridge 1998).

Studies examining the relationship between BCAA supplementation and exercise-induced fatigue have produced equivocal results (for review, see Meeusen et al. 2006), possibly related to methodologic differences in timing and dosages of BCAA supplementation. Exercise performance is often unaltered, but ratings of perceived exertion, mood states, or cognitive function during exercise may be improved after BCAA supplementation (Blomstrand et al. 1997, 1991; Jakeman 1998). There are limited data on the BCAA effects on intermittent high-intensity exercise performance (Davis et al.
1999) and mental performance of team sport athletes (Blomstrand et al. 1991), and most studies have focused on continuous exercise protocols (e.g., cycling time to exhaustion), rather than the motor skill performance or decision making characteristic of team sports.

The aim of this study was to investigate the effect of a high-dose LNAA supplement ingested $3 \mathrm{~h}$ prior to high-intensity intermittent exercise on reactive motor skill, agility, and cognitive performance in Australian Rules Football (ARF) players.

\section{Materials and methods}

\section{Participants}

Fifteen subelite male ARF players were recruited from local football clubs (Table 1). Prior to participation, all players provided informed written consent. Two players withdrew from the study without completing all testing procedures because of injuries sustained in unrelated games, and their data were not included in any analysis. The study design and procedures met the National Health and Medical Research Council guidelines for research using human participants by the Australian Institute of Sport Ethics Committee; the Standing Committee on Ethical Research in Humans, Monash University; and the Human Research Ethics Committee of Victoria University.

\section{Study design}

The study was conducted over 5 sessions during consecutive weeks, and each session was conducted at the same time of day. During the first 2 sessions, players completed exercise test familiarization, anthropometric measures, and fitness testing. All players completed an initial baseline (BL) trial of reactive motor skills testing (RMST) and reactive agility (RAT) testing, in which no protein supplementation was given. In the final 2 sessions, players completed a double-blind crossover (randomized and counterbalanced) trial, receiving either the tryptophan-"depleting" (without tryptophan) or protein control (PC; with tryptophan) mixtures of LNAA to decrease or maintain brain serotonin levels, respectively.

\section{Preliminary testing and familiarization}

Players were measured for height, body mass, and fat (sum of 7 skinfolds) at least $2 \mathrm{~h}$ postprandial and in a wellhydrated state (Table 1). Players then completed 2 familiarization sessions on separate days of the sports-specific RMST, RAT, and psychological questionnaires. Session 1 testing was followed by the 20-m multistage shuttle run test for estimation of maximal heart rate (HRmax) and maximal oxygen consumption. After the second session, players completed 3 repetitions of the $20-\mathrm{m}$ sprint test, with the fastest sprint recorded as the criterion time.

\section{Dietary and exercise control}

All players maintained their normal diet in the 3 days leading up to each session and were instructed to replicate this food intake before each trial. Players were required to abstain from alcohol and caffeinated products for $24 \mathrm{~h}$ prior to and on the day of testing. Players were also asked to engage in a moderate-intensity (1-h) training session $24 \mathrm{~h}$ prior to the testing sessions, allowing them to maintain their in-season 
Table 1. Subject characteristics $(n=13)$.

\begin{tabular}{ll}
\hline Characteristic & Mean \pm SD \\
\hline Age $(\mathrm{y})$ & $22 \pm 3$ \\
Height $(\mathrm{m})$ & $1.79 \pm 0.08$ \\
Mass $(\mathrm{kg})$ & $83.1 \pm 17.3$ \\
Sum of 7 skinfolds $(\mathrm{mm})$ & $96 \pm 46$ \\
$\mathrm{HRmax}\left(\right.$ beats $\left.\cdot \mathrm{min}^{-1}\right)$ & $197 \pm 7$ \\
Estimated $\dot{V} \mathrm{O}_{2 \max }\left(\mathrm{mL} \cdot \mathrm{kg}^{-1} \cdot \mathrm{min}^{-1}\right)$ & $50.3 \pm 8.8$ \\
20-m sprint time (s) & $3.17 \pm 0.23$ \\
\hline
\end{tabular}

Note HRmax, maximal heart rate achieved in 20-m shuttle run; $\dot{V} \mathrm{O}_{2 \text { max }}$, maximal oxygen consumption.

training programs, with further instructions to replicate this training before every testing day.

\section{BL trial}

Players returned to the testing venue 7 days later, approximately $3.5 \mathrm{~h}$ prior to the testing session, having not eaten for the previous $2 \mathrm{~h}$. A low-glycemic-index (GI) high-carbohydrate meal was consumed $3 \mathrm{~h}$ prior to exercise testing. Twenty minutes before the warm-up, players completed 2 standard paper-based psychological tests to assess mood states and cognitive function. Ten minutes before the commencement of the warm-up exercise, participants consumed $600 \mathrm{~mL}$ of a commercial sports drink (Gatorade Fierce Berry instant mix, $8 \% \mathrm{w} / v$ carbohydrate solution). The sports drink consumption was confirmed by the experimenters. After the standardized warm-up, participants undertook the RMST and RAT before completing $30 \mathrm{~min}$ of the fatiguing exercise. Immediately upon completion of the exercise bout, participants were retested with the RMST, RAT, and psychological tests.

\section{Supplementation trials}

Players again reported to the testing venue $3.5 \mathrm{~h}$ prior to the testing session, and followed the same dietary and testing schedule as above. However, the PC or depleting supplements were ingested immediately before the meal in a doubleblind, counterbalanced, cross-over design. Serial blood samples were taken immediately before the meal and protein supplementation, $3 \mathrm{~h}$ postsupplementation, and immediately after the fatiguing exercise.

\section{Skills and decision testing}

RMST was conducted using the SmartSpeed lightgate system (Fusion Sport, Brisbane, Queensland, Australia) to assess players' skills in a reactive setting. Players were instructed to complete the test as quickly and as accurately as possible. The test was conducted on the Y-shaped 20-m testing area (Fig. 1). The test required the player to cross the starting gate, collect a stationary ball, progress past a second set of gates, causing one of the 2 final gates to randomly give a light signal, to which the player responded by handballing the football to the target (scored) and then proceeding through the final gate, stopping the timer (Fig. 1). Each player performed 12 repetitions of this test with a 60 -s recovery, randomized to ensure the completion of 6 left and 6 right breaks. The test times were recorded using Smartspeed software on a linked personal digital assistant.

The accuracy-precision of the skill (handballing) performed was determined by manually recording the score of each repetition achieved: 0 for missed or frame hit, 1 point for ball contact on the wooden target (shaded area in Fig. 1), and 2 points for the ball passing through the central $75-\mathrm{cm}$ diameter hole in the target. The targets were designed such that the central hole was the equivalent of placing the handball level with the chest of a 1.85-m-tall player. Because this test was designed to quantify reactive skill performance, there was always the potential for players to trade speed for accuracy. To account for this potential shortcoming, we calculated a test performance index (TPI), computed as the repetition score divided by the repetition time $\left(\operatorname{score}^{-1}\right)$. In a pilot study $(n=25)$ (N. Gregory, N. Stepto, and J. Keating, unpublished data) using the RMST, we observed substantial variability in the initial repetitions (1-5), so only repetitions 6-12 were used for time, score, and TPI data. The typical error (or coefficient of variation $(\mathrm{CV})$ ) for test-retest reliability for repetitions 6-12 of participants in this study was $3.7 \%$ (90\% confidence interval (CI): $2.8 \%-5.6 \%$ ).

The RAT was timed using the SmartSpeed lightgate system and was conducted as described previously (Farrow et al. 2005). The typical error for test-retest reliability for the subject's repetition time in this study was $2.7 \%$ (90\% CI, $2.0 \%-4.0 \%)$.

\section{Amino acid supplements}

In each experimental trial, players ingested one of the 2 protein drinks in randomized order immediately before the pre-exercise meal $3 \mathrm{~h}$ prior to the exercise trials. The protein mixture was provided as a suspension of the pure amino acids (either the tryptophan depleting or the PC) in $250 \mathrm{~mL}$ unsweetened orange juice plus $600 \mathrm{~mL}$ water, and was consumed in the presence of the investigators. The individual BCAA powders, except for tryptophan, were obtained from Musashi (Nestle Nutrition, Knotting Hill, Victoria, Australia) and were weighed and combined to form either the tryptophan and therefore serotonin-"depleting" mixture (43.0 g; $13.5 \mathrm{~g}$ leucine, $8 \mathrm{~g}$ isoleucine, $8.9 \mathrm{~g}$ valine, $5.7 \mathrm{~g}$ phenylalanine, and $6.9 \mathrm{~g}$ tyrosine) or the PC mixture (45.3 g; $13.5 \mathrm{~g}$ leucine, $8 \mathrm{~g}$ isoleucine, $8.9 \mathrm{~g}$ valine, $5.7 \mathrm{~g}$ phenylalanine, $6.9 \mathrm{~g}$ tyrosine, and $2.3 \mathrm{~g}$ tryptophan (USP; Nationwide Compounding Pharmacy; Caulfield, Australia)). These supplements are based on the amino acid mixtures that lower serotonin production and alter behavior in humans (Moore et al. 2000; Nathan et al. 2004; Nishizawa et al. 1997). These amino acid supplementation regimens were generally well tolerated, and only 5 of 13 participants reporting feelings of mild lethargy and (or) bloating.

The pre-exercise meal consisted of a low-GI high-carbohydrate meal (a 300-g salad sandwich; $1054 \mathrm{~kJ}, 71 \%$ carbohydrate, $7 \%$ fat, $14 \%$ protein). Players then rested at the venue until testing began. When players began the warm-up, they consumed $600 \mathrm{~mL}$ of a commercially available sports drink (Gatorade). The pre-exercise meal was provided to reflect a more practical sports setting, but we acknowledge that the meal is likely to have influenced plasma LNAA concentrations and the free tryptophan/LNAA ratio (Davis et al. 1992). However, we believe this effect is likely to be minimal because this feeding protocol was used in all experimental and BL trials. 
Fig. 1. Schema of test area set-up (A) and testing procedure (B) for the reactive motor skills test (RMST). The test required the player to cross the starting gate, collect a stationary ball (B, panel 1), progress past a second set of gates (B, panel 2), causing one of the 2 final gates to randomly give a light signal, to which the player responded by handballing the football to the target (scored) (B, panel 3$)$ and then proceeding through the final gate, stopping the timer (B, panel 4).

A

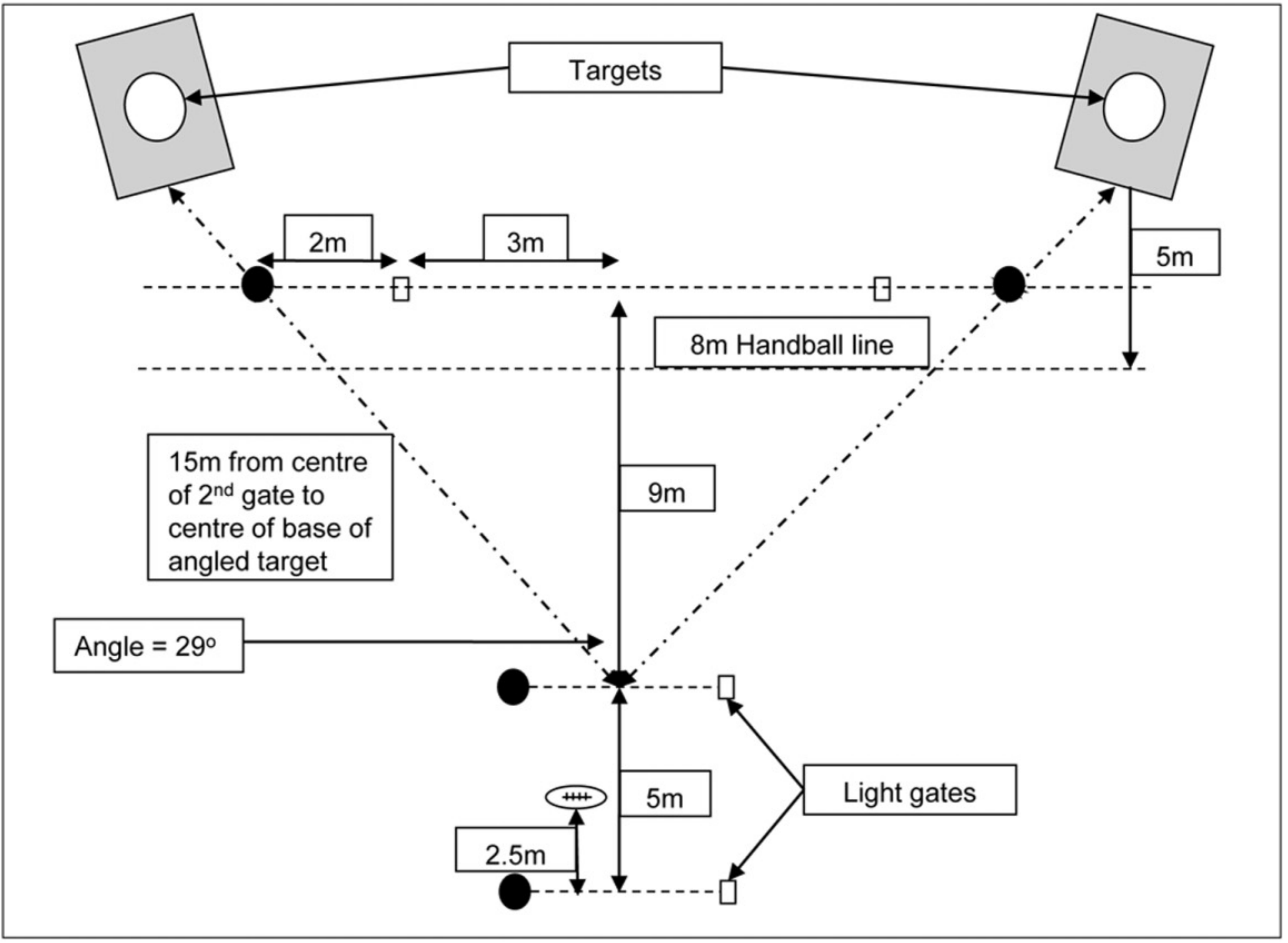

B

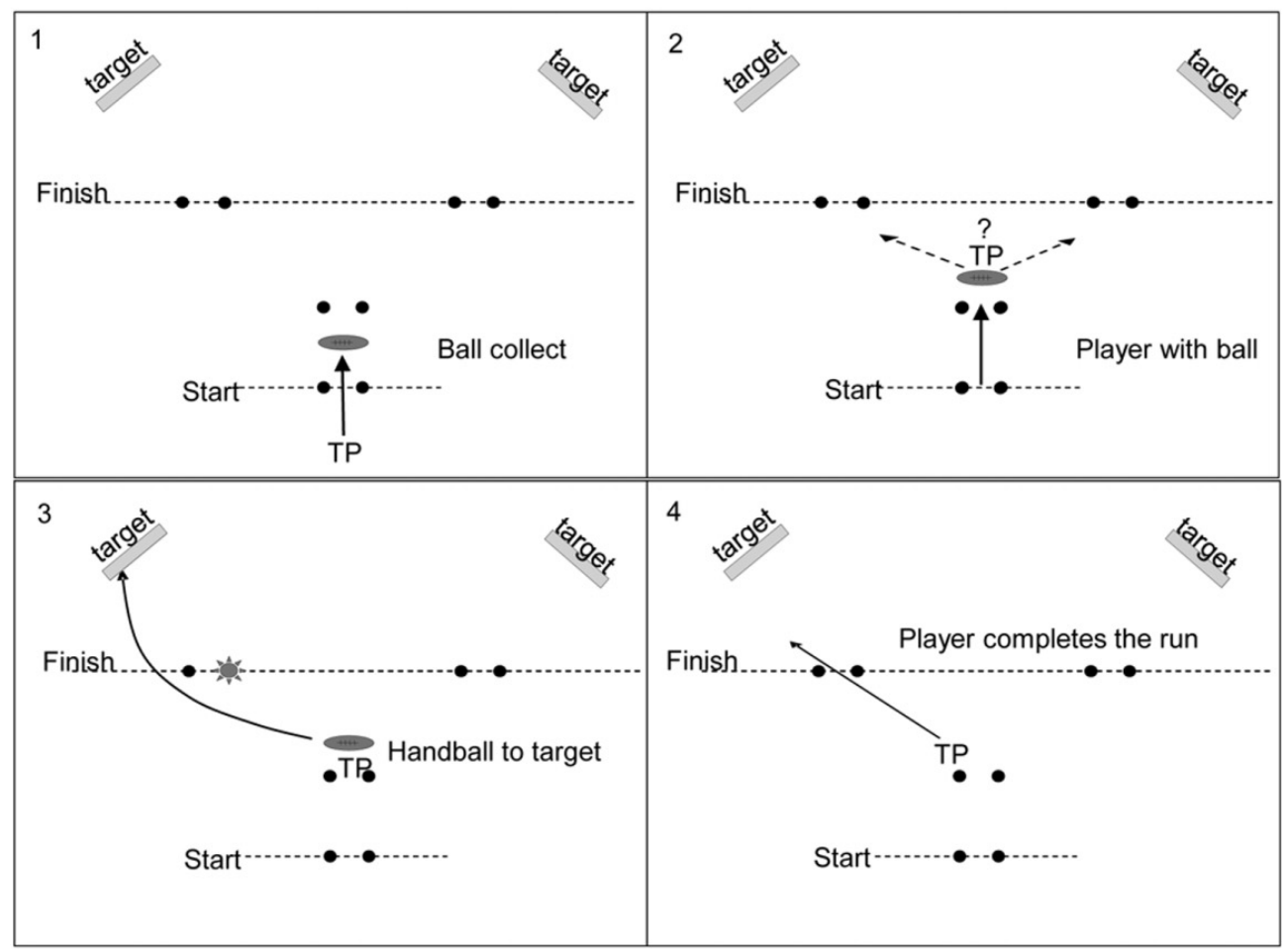

Key: TP - test player

light gate

\section{Fatiguing exercise protocol}

The 30-min fatiguing exercise involved a modification of the Yo-Yo intermittent endurance test (Level 1; Bangsbo Sport, Copenhagen, Denmark). Players were given a football 
to bounce once every lap while completing the entire Yo-Yo endurance test. Players were allowed to sit out a maximum of 2 consecutive return runs for additional recovery (recorded and subtracted for total distance of the test), but had to re-enter the test at the next shuttle. Players' heart rates were monitored and recorded using a Polar RS400 heart rate monitor (Pursuit Performance, Adelaide, South Australia, Australia).

\section{Blood sampling and analysis}

Blood samples (4 mL each) were collected via a sterile valve and in-dwelling cannula inserted in an antecubital vein kept patent with sterile saline $(0.9 \%$; Astra Zeneca, North Ryde, New South Whales, Australia). The blood was placed in lithium heparin vacutainers (Interpath Services, Heidelberg West, Victoria, Australia) and centrifuged at $1500 \mathrm{~g}$ for $15 \mathrm{~min}$. Each plasma sample was collected, aliquoted into separate microfuge tubes for each analysis, and stored at $-20{ }^{\circ} \mathrm{C}$.

Plasma glucose and lactate were analyzed on the YSI STAT2000 automated analyzer (Yellow Springs Instruments, Yellow Springs, Ohio, USA). Plasma insulin was determined using an enzyme immunoassay (DakoCytomation UK No. K6219; Dako, Cambellfield, Victoria, Australia); $25 \mu \mathrm{L}$ of plasma was pippetted into duplicate wells on a 96-well plate and processed according to the manufacturer's instructions. The limit of detection was $3 \mathrm{pmol} \cdot \mathrm{L}^{-1}$ with an intra- and interassay variability of $7.8 \%$ and $10.4 \%$, respectively.

Concentrations of the free amino acids tryptophan, tyrosine, phenylalanine, valine, leucine, and isoleucine in plasma were determined from a modified protocol (Nathan et al. 2004) using precolumn derivatization with 6-aminoquinolyl$\mathrm{N}$-hydroxysuccinimidyl carbamate (AQC) and quantification by reversed-phase high-performance liquid chromatography. All amino acids were detected by ultraviolet absorbance. Briefly, plasma samples $(100 \mu \mathrm{L})$ were diluted 1:1 with internal standard (norvaline) solution and deproteinized by ultrafiltration through a membrane with a $10-\mathrm{kDa}$ nominal molecular mass cut-off (Microcon Ultracel YM-10, Millipore, Bedford, Mass., USA). The filtrate $(20 \mu \mathrm{L})$ was then subjected to AQC derivatization and analysis on an ACQUITY Ultra Performance LC system containing a BEH C18 1.7- $\mu \mathrm{m}$ column (Waters Corporation, Milford, Mass., USA) with detection at $260 \mathrm{~nm}$ and a flow rate of $0.7 \mathrm{~mL} \cdot \mathrm{min}^{-1}$. The limit of quantitation was $0.5 \mu \mathrm{mol} \cdot \mathrm{L}^{-1}$ with intra- and interassay variability of $4.5 \%$ and $5.1 \%$, respectively, for tryptophan, and $<2.3 \%$ and $3.8 \%$, respectively, for the other LNAA.

\section{Psychological testing}

Over the duration of the study, players' cognitive function and mood states were analyzed using standard questionnaires.

Cognitive functioning was analyzed using the Stroop Color-Word test, which required participants to read as many words, colours, or coloured words as possible in $45 \mathrm{~s}$. This test has been used to measure an untrained healthy individual's ability to resist cognitive interference (Blomstrand et al. 1991; Young et al. 1985). These tests were administered $20 \mathrm{~min}$ before, and immediately after, exercise testing in weeks 3 to 5 .

Change in mood was analyzed using Profile of Mood State (POMS) questionnaires. Sixty-five questions were used to measure different metrics of mood, including fatigue, vigor, confusion, depression, anger, and tension. Because mood is quite variable, this questionnaire is designed to quantify changes that occur over time (Young et al. 1985). These questionnaires were administered $20 \mathrm{~min}$ before, and immediately after, exercise testing in weeks 3 to 5 .

\section{Statistical analysis}

Data are expressed as mean \pm SD. All data, except POMS and Stroop scores, were log transformed to reduce bias due to nonuniformity of error. All blood parameters and TPI were analyzed using 2-way repeated-measures analysis of variance (ANOVA), and where significance was shown, data were subjected to a Bonferroni post hoc test. A one-way repeated-measures ANOVA with Tukey's post hoc analysis was used to identify significant time effects. Significance was accepted at $p<0.05$. We also used the effect size (ES) statistic with a $90 \%$ CI to determine the magnitude of the effects and the precision of the estimation (Batterham and Hopkins 2006; Hopkins 2000). Performance data were analyzed at the postonly time point, because performing an analysis of a pre-post parallel-groups controlled trial using only the postintervention values produced estimates with better precision, given the degree of background noise in the dependent variables (Batterham and Hopkins 2006; Hopkins 2000). In contrast, we used a pre-post analysis to analyze the effect of LNAA on the concentration of plasma parameters. Magnitudes of change were classified as substantial when there was a $\geq 75 \%$ likelihood of the effect being equal to or greater than the smallest worthwhile change. The smallest worthwhile change in performance tests and physiological measures was estimated as $0.2 \times$ between-subject $\mathrm{SD}$ and was classified as trivial $(\mathrm{ES}<0.2)$, small $(0.2-0.6)$, moderate $(0.6-1.2)$, large (1.2-2.0), or very large $(>2.0)$. Effects were reported as unclear when the $\pm 90 \%$ CI spanned both substantial positive (worthwhile) and negative thresholds. Reliability of the RAT and RMST were determined as typical error expressed as a CV (Hopkins 2000).

\section{Results}

\section{RAT and RMST performance}

First, we established that our fatiguing exercise protocol substantially impaired agility (RAT) and motor skill (RMST) performance (Fig. 2A, 2C, and 2E). The exercise protocol (without any protein supplementation) induced a moderate increase (slowing) of the RAT mean repetition time of $5.7 \% \pm 3.0 \%$ (mean $\pm 90 \% \mathrm{CI}$ ). Similarly, in the RMST, the mean repetition was moderately increased by $6.0 \% \pm 3.0 \%$. This decrement in repeat performance was accompanied by significant reduction in the TPI $(-16 \% \pm 14 \%$; ES $-0.79 \pm$ $0.60 ; p=0.03$; Fig. 3$)$ and a moderate $(11 \% \pm 12 \%)$ reduction in the accuracy of the handball task.

The effects of the LNAA mixtures on the performance of the RAT and RMST are shown in Fig. 2. Depleting serotonin moderately improved the RAT performance by $-5.2 \% \pm 3.4 \%$ (Fig. 2B) compared with the BL trial, after fatiguing exercise. Similarly, PC elicited a small improvement in mean RAT repetition time $(-2.9 \% \pm 4.1 \%$; Fig. $2 \mathrm{~B})$. The difference between the depleting and $\mathrm{PC}$ treatments on the mean repetition performance time of the RAT was trivial (Fig. 2B). The 2 out- 
Fig. 2. The mean repetition (6-12) time and score results of the exercise performance tests conducted before and after a fatiguing exercise protocol in Australian Rules Football players after taking either no supplement (baseline), a serotonin-depleting supplement, or a protein control supplement. (A) Change in mean total repetition time of the baseline reactive agility test (RAT). (B) Mean repetition time in RAT after fatiguing exercise on the different large neutral amino acid supplementation. (C) Change in mean total time of the baseline reactive motor skills test (RMST) before and after exercise. (D) Mean total times after exercise of the RMST in all supplementation groups. (E) Change in mean score achieved for the handball for the baseline RMST before and after exercise. (F) Mean score achieved for the handball in the RMST for all supplementation trials. Data are means \pm SD for $n=13$; Pre Ex, pre-exercise; Post Ex, postexercise; BL, baseline with no protein supplement; Dep, tryptophan-serotonin depleting; PC, protein control. *, Moderate difference between pre- and postexercise means; $\dagger$, small difference between treatments means; $\ddagger$, a moderate difference between treatments means.
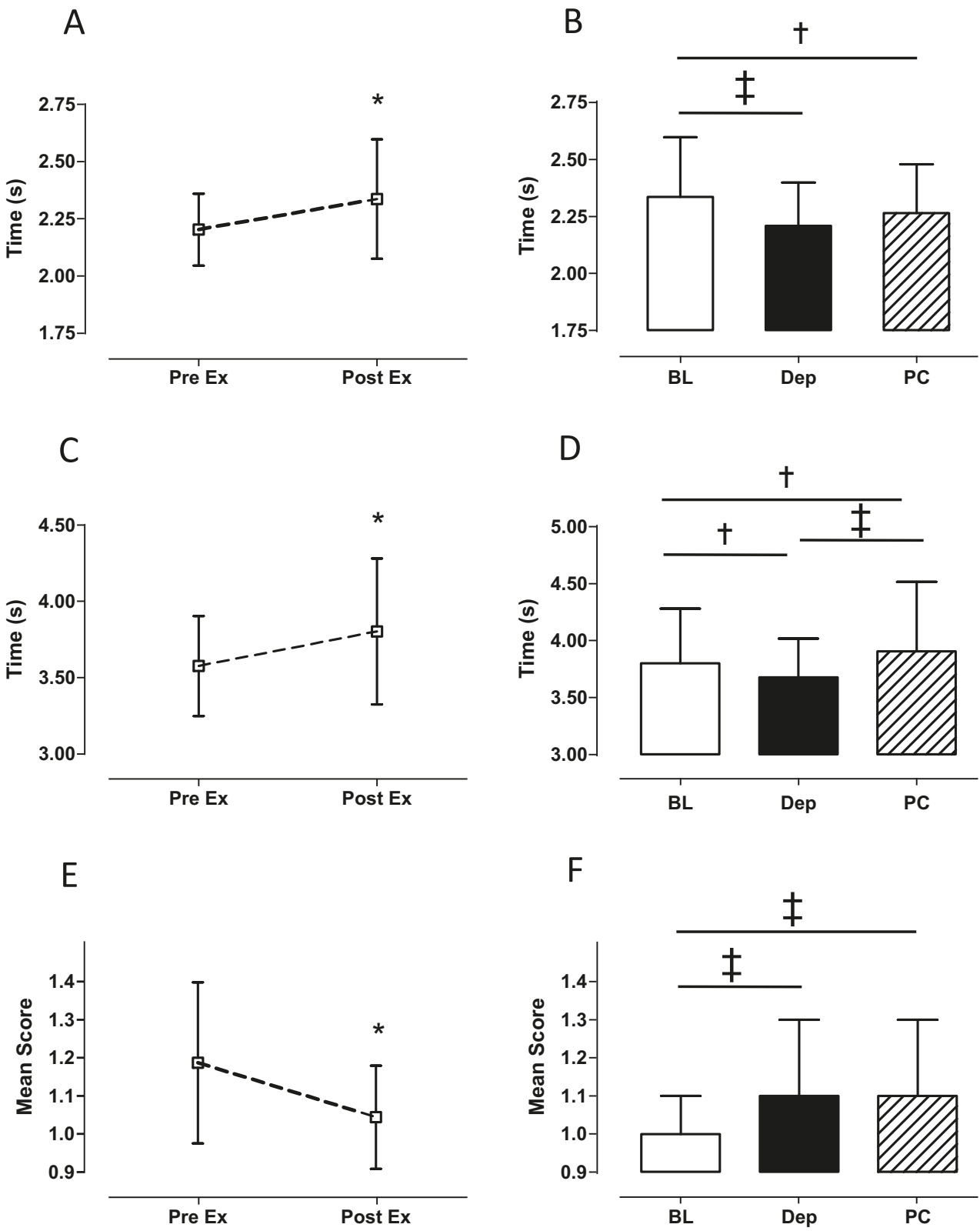

come measures of the RMST are presented as a mean time for each repetition and a mean score for each handball in Fig. 2D and 2F, respectively. Clearly, serotonin depleting and PC had a divergent effect on the RMST mean repetition time after fatiguing exercise. Depletion elicited a small improvement $(-3.0 \% \pm 2.7 \%$, ES $-0.25 \pm 0.21)$ in contrast to the small decrement in RMST performance $(2.4 \% \pm 2.7 \%$, ES $0.20 \pm 0.21$ ) of the PC, compared with the BL trial. Serotonin depletion elicited a moderate improvement in repeti-

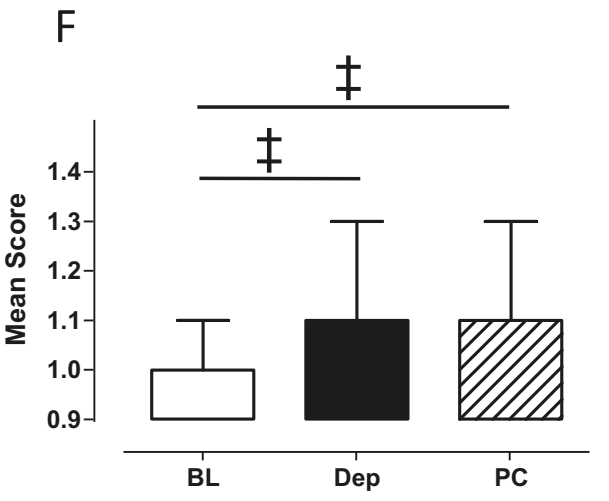

tion performance time postexercise when compared with PC $(-5.3 \% \pm 4.3 \%, \mathrm{ES}-0.43 \pm 0.33)$.

Skill performance was moderately improved after exercise, independent of the supplementation treatment, when compared with the BL test $(7.4 \% \pm 9.8 \%$ and $8.0 \% \pm 9.5 \%$ for serotonin depletion and PC, respectively). However, the TPI, which accounted for the speed-accuracy trade-off, decreased with fatiguing exercise, with the greatest decrements in the $\mathrm{BL}$ and $\mathrm{PC}$ trials $(-16 \% \pm 14 \%, \mathrm{ES}-0.83 \pm 0.58, p=0.03$; 
Fig. 3. The mean repetition TPI (TPI = repetition score/total repetition time (s)) for the reactive motor skills test (RMST) conducted before and after a fatiguing exercise protocol in Australian Rules Football players after taking either no supplement (baseline), a serotonin-depleting supplement, or a protein control supplement. Data are presented as means \pm SD for $n=13$. TPI, test performance index; BL, baseline with no protein supplement; Dep, tryptophan-serotonin depleting; PC, protein control. *, Small difference between means; $\dagger$, significant difference between means $(p<0.05)$.

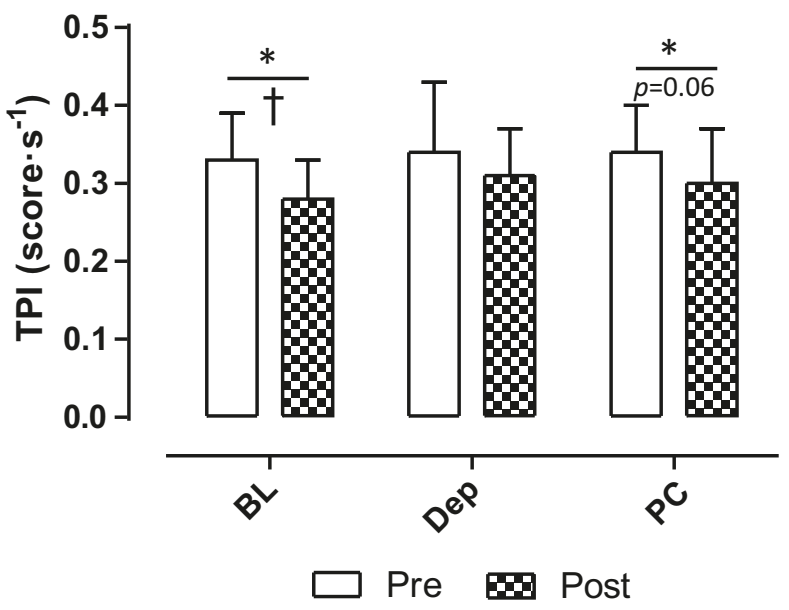

and $-13 \% \pm 12 \%, \mathrm{ES}-0.65 \pm 0.54, p=0.06$, respectively; Fig. 3), compared with the depleting trial $(-5.1 \% \pm 14.8 \%$, $\mathrm{ES}-0.27 \pm 0.64)$. The exercise-induced decrements in TPI were $11.4 \%$ and $7.9 \%$ greater in the $\mathrm{BL}$ and $\mathrm{PC}$ trials, respectively, compared with the depleting trial, but the magnitudes of these changes were unclear.

\section{Fatiguing exercise protocol}

There were no substantial differences between the experimental and control trials in cardiovascular responses and distance covered in the fatiguing exercise protocol. Heart rate, $\%$ HRmax, and distance covered during the fatiguing exercise bout were similar in the 3 trials (Table 2). There were trivial differences in the distance covered between serotonin depletion and BL during the fatiguing exercise protocols (Table 2). Similarly, any differences in mean heart rate and \%HRmax during exercise in the PC trial compared with the BL trial were trivial (Table 2).

\section{Mood states and cognitive function}

The fatiguing exercise bout increased the total mood disturbance scores $(5.6 \pm 5.4$ raw score (ES $0.31 \pm 0.30)$; moderate effect; Table 3$)$ and the fatigue score $(3.7 \pm 1.6$ raw score (ES $1.48 \pm 0.64$ ); large effect; Table 3) at BL. During the depleting trial, exercise elicited larger changes in total mood disturbance $(11.5 \pm 12.2$ raw score (ES $0.64 \pm 0.68)$ ) but similar changes in fatigue scales $(3.8 \pm 2.2$ raw score (ES $1.54 \pm 0.9)$ ), compared with BL. The depleting trial elicited a small increase in the vigor score (Table 3). Similarly, the effect of the exercise on increasing total mood disturbance $(13.0 \pm 4.9$ raw score (ES $0.72 \pm 0.27))$ and fatigue $(5.0 \pm$ 2.3 raw score (ES $2.0 \pm 0.93)$ ) and decreasing vigor $(-5.0 \pm$ 2.3 raw score $(\mathrm{ES}-0.63 \pm 0.29)$ ) were moderate, large, and moderate in PC, respectively (Table 3 ).

The effect of fatiguing exercise on the Stroop scores only became apparent during the depletion trial, in which Stroop Color-Word scores $(10.2 \pm 11.8$ raw score (ES $0.64 \pm$ 0.74); moderate effect) and Color scores (18.8 \pm 19.9 raw score (ES $0.63 \pm 0.67$ ); moderate effect) improved after exercise (Table 3). When comparing the postexercise scores across the 3 trials, depletion resulted in a moderate improvement in the hardest Stroop scale score (Color-Word score; $11 \pm 10$ raw score $(\operatorname{ES} 0.5 \pm 0.5))$ compared with the BL trial. However, this improvement in scores was unclear when comparing the depleting trial with the PC trial, despite the $9 \pm 13($ ES $0.4 \pm 0.6)$ score difference postexercise.

\section{Blood parameters}

Plasma glucose, insulin, and lactate concentrations were altered over the time course of the trials in response to supplementation and (or) exercise, but there were no differences between the 2 protein treatments. Plasma lactate concentration remained at $1.4 \pm 0.4 \mathrm{mmol} \cdot \mathrm{L}^{-1}$ during the resting period but increased to $5.4 \pm 0.9 \mathrm{mmol} \cdot \mathrm{L}^{-1}$ immediately after exercise in all trials (mean \pm SD). Plasma glucose remained at $3.6 \pm 0.6 \mathrm{mmol} \cdot \mathrm{L}^{-1}$ during rest and increased to $4.7 \pm$ $1.0 \mathrm{mmol} \cdot \mathrm{L}^{-1}$ after exercise. Plasma insulin was $60 \pm$ $19 \mathrm{pmol} \cdot \mathrm{L}^{-1}$ prior to protein supplementation, increased to $156 \pm 58 \mathrm{pmol} \cdot \mathrm{L}^{-1}$ immediately before exercise, and then decreased to $86 \pm 45 \mathrm{pmol} \cdot \mathrm{L}^{-1}$ immediately postexercise.

Plasma LNAA concentrations are displayed in Table 4. The LNAA concentrations quantified in the plasma, including isoleucine, leucine, phenylalanine, tyrosine, and valine, and the total LNAA content were similar for the 2 protein trials at all time points. However, the free tryptophan concentrations diverged both immediately pre- $\left(2 \pm 1 \mu \mathrm{mol} \cdot \mathrm{L}^{-1} \mathrm{vs}\right.$. $\left.15 \pm 9 \mu \mathrm{mol} \cdot \mathrm{L}^{-1}\right)$ and postexercise $\left(4 \pm 3 \mu \mathrm{mol} \cdot \mathrm{L}^{-1}\right.$ vs. $15 \pm$ $\left.8 \mu \mathrm{mol} \cdot \mathrm{L}^{-1}\right)$, reflecting the absence or presence of the tryptophan in the depleting and PC mixtures, respectively (Table 4). Because the biological effectiveness of the treatments depends on competitive inhibition of the LNAA transporter uptake of the tryptophan, the ratio of free tryptophan to total free LNAA is the key measure (Fig. 4). Clearly, the presence of tryptophan in PC resulted in a greater free tryptophan/ LNAA ratio, both immediately pre- and postexercise (Fig. 4). The ratio changed from $\sim 0.0045$ at rest in both trials to $0.0071 \pm 0.0046$ and $0.0105 \pm 0.0058$ in the PC treatment and $0.0007 \pm 0.0007$ and $0.0029 \pm 0.0017$ in the depleting treatment immediately pre- and postexercise, respectively. The ratio between PC and depleting treatments was substantially increased from rest to pre-exercise $(920 \% \pm 48 \%$; large; $p<0.01)$. Subsequent changes in the ratio from pre- to postexercise were much smaller $(-64 \% \pm 45 \%$; moderate; $p<$ $0.05)$ in PC compared with the depleting treatment. This difference was evident despite the absolute postexercise ratios being $326 \% \pm 43 \%$ (moderate; $p<0.05$ ) greater in PC vs. depleting treatments (Fig. 4)

\section{Discussion}

In this study we demonstrate, to our knowledge for the first time, that consumption of a large quantity of LNAA $3 \mathrm{~h}$ prior to exercise, which depletes brain tryptophan supply and therefore serotonin production, delayed or reduced the impact of exercise-induced fatigue on player reactive agility (by $\sim 5 \%$ ) and skill performance (by $\sim 3 \%$ for time, $\sim 8 \%$ for 
Table 2. Performance and exercise intensity measures during the fatiguing exercise protocol for baseline (BL), serotonin-depleting (Depleting), and protein control (PC) trials.

\begin{tabular}{llll}
\hline Parameter & BL & Depleting & PC \\
\hline Distance $(\mathrm{km})$ & $4.0 \pm 0.7$ & $4.1 \pm 0.7$ & $4.0 \pm 0.7$ \\
HRmax during exercise (beats· $\mathrm{min}^{-1}$ ) & $190 \pm 6$ & $189 \pm 5$ & $187 \pm 8$ \\
Mean HR during exercise (beats· $\mathrm{min}^{-1}$ ) & $168 \pm 8$ & $168 \pm 6$ & $167 \pm 8$ \\
Mean HR as \%HRmax (\%) & $86 \pm 5$ & $86 \pm 4$ & $85 \pm 4$ \\
\hline
\end{tabular}

Note: Data are presented as means $\pm \mathrm{SD}$ for $n=10$ subjects for HR data and $n=13$ subjects for distance covered. There were no substantial differences between any variables. HR, heart rate; HRmax, maximal heart rate.

Table 3. Scores from the Stroop and POMS psychological questionnaires.

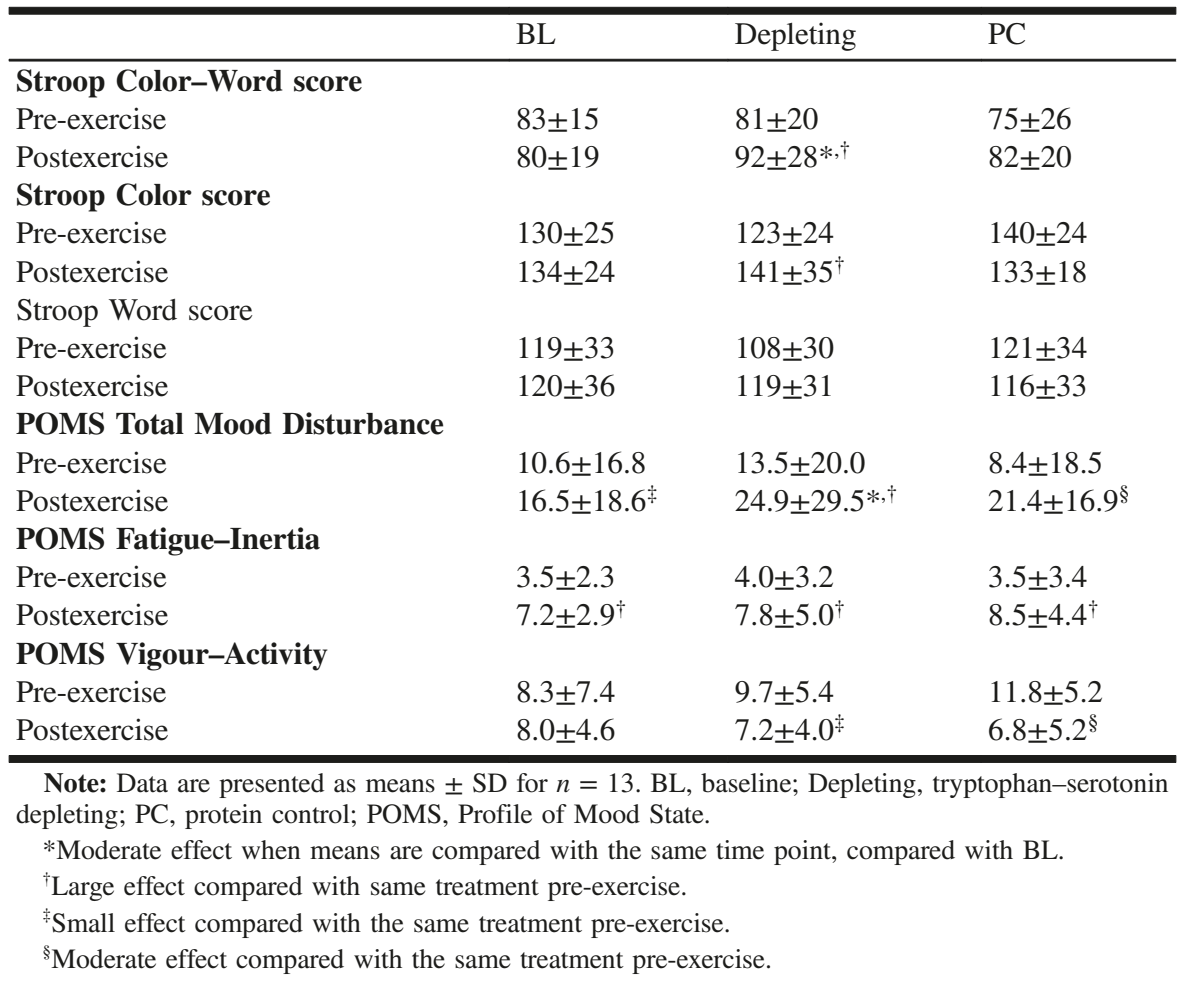

score, and $\sim 10 \%$ for TPI). These findings indicate that neurotransmitters, in particular serotonin, play a role in the central component of exercise fatigue and can impair skill-based sporting tasks. Novel supplementation strategies with amino acids offer a practical means of enhancing decision making and skill performance in team and skill-based sports.

The repeat-effort exercise protocol elicited moderate decrements in performance of reactive agility and skills tasks (time, accuracy, and TPI) in ARF players in the presence of carbohydrate supplementation. Our results indicate that the testing protocols were effective in detecting fatigue-induced changes in the reactive skill and agility performance of the players. Furthermore, we established that any substantial changes in exercise test performance among the 3 trials could be attributed to the protein supplements because there were no differences in the amount or intensity of exercise performed during the fatiguing exercise protocols (Table 2).

We demonstrated that high-dose LNAA (which includes BCAA) supplementation attenuated the fatigue-induced dec- rements in performance time of RMST and RAT (Fig. 2 and Fig. 3). The attenuated loss of performance was evident after the supplementation designed to deplete tryptophan and serotonin (Pardridge 1998), as was demonstrated in the increased plasma LNAA concentrations and divergent free tryptophan/ LNAA ratio between the depleting and PC trials (Table 4 and Fig. 4). This finding is in contrast to those of most other studies using amino acid supplements, which failed to delay the onset of fatigue in timed exercise tasks (Blomstrand et al. 1997; Davis et al. 1999; Watson et al. 2004; Weicker and Struder 2001). The lack of amino acid influence on exercise performance in these studies could be explained by differences in methodological approaches to supplementation. Most exercise studies have supplied only small quantities of BCAA (7-20 g) compared with the $30.4 \mathrm{~g}$ (or $\sim 44 \mathrm{~g}$ LNAA) used in this study. Our approach is based on previous work (Moore et al. 2000; Nathan et al. 2004; Nishizawa et al. 1997) but utilized only LNAA that were commercially available as supplements. This resulted in an LNAA mixture quantity of $<100 \mathrm{~g}$ but containing enough LNAA to 
Table 4. Plasma amino acid concentration of selected free LNAA before, $3 \mathrm{~h}$ postsupplementation, and immediately postexercise.

\begin{tabular}{llll}
\hline $\begin{array}{l}\text { Amino acid } \\
\text { concentration } \\
\left(\mu \text { mol· } L^{-1}\right)\end{array}$ & Rest & Pre-exercise & Postexercise \\
\hline $\begin{array}{l}\text { Isoleucine } \\
\text { Depleting }\end{array}$ & $85 \pm 25$ & $366 \pm 100$ & $202 \pm 98$ \\
PC & $67 \pm 15$ & $326 \pm 120$ & $177 \pm 115$ \\
Leucine & & & \\
Depleting & $147 \pm 44$ & $578 \pm 129$ & $366 \pm 174$ \\
PC & $143 \pm 32$ & $574 \pm 97$ & $320 \pm 166$ \\
Phenylalanine & & & \\
$\begin{array}{l}\text { Depleting } \\
\text { PC }\end{array}$ & $68 \pm 24$ & $174 \pm 43$ & $147 \pm 52$ \\
Tryptophan* & $65 \pm 22$ & $145 \pm 39$ & $124 \pm 39$ \\
$\begin{array}{l}\text { Depleting } \\
\text { PC }\end{array}$ & $3 \pm 1$ & $2 \pm 1$ & $4 \pm 3$ \\
Tyrosine & $3 \pm 1$ & $15 \pm 9^{\dagger}$ & $15 \pm 8^{\dagger}$ \\
Depleting & $83 \pm 19$ & $214 \pm 40$ & $232 \pm 63$ \\
PC & $76 \pm 24$ & $200 \pm 51$ & $233 \pm 55$ \\
Valine & & & \\
Depleting & $241 \pm 35$ & $935 \pm 167$ & $614 \pm 190$ \\
PC & & $903 \pm 124$ & $600 \pm 245$ \\
Total LNAA & $225 \pm 17$ & & \\
Depleting & $623 \pm 112$ & $2268 \pm 330$ & $1561 \pm 535$ \\
PC & $577 \pm 55$ & $2149 \pm 266$ & $1453 \pm 563$ \\
\hline
\end{tabular}

Note: Data are presented as means \pm SD for $n=10$. LNAA, large neutral amino acids; Depleting, trypthophan-serotonin depleting; PC, protein control.

* Free unbound tryptophan reported, but a large amount of plasma tryptophan is bound to plasma albumin and not measured in this study (Pardridge 1998).

$\dagger$ Very large differences in means at the same time point in depleting trial $(p<0.05)$.

¥arge differences in means at the same time point in depleting trial $(p<0.01)$

Fig. 4. Free tryptophan/total LNAA ratio demonstrating the potential competitive inhibition of the LNAA transporter at the blood-brain barrier. Data are presented as means \pm SD for $n=10$. LNAA, large neutral amino acid; Dep, tryptophan-serotonin depletion; PC, protein control; Rest, resting; Pre Ex, pre-exercise; Post Ex, postexercise. *, Very large difference between the same time points in depleting and loading trials; $\uparrow$, moderate difference between the same time points in depleting and loading trials.
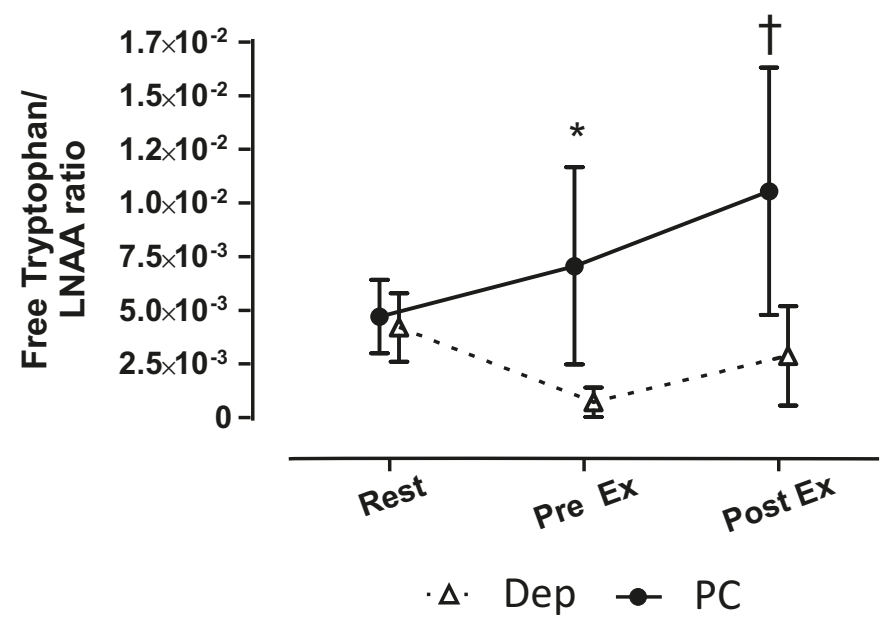

selectively deplete brain tryptophan and promote dopamine production. Secondly, the timing of the BCAA supplementation with or without carbohydrate was never more than 120 min prior to exercise, and may have been short enough to blunt the competitive inhibition of LNAA transporters and reduce serotonin production (Nathan et al. 2004). A further consideration is that all existing studies have investigated BCAA effects in constant-load closed-loop exercise tasks, including cycling (Blomstrand et al. 1997; Watson et al. 2004; Weicker and Struder 2001) and shuttle running (Davis et al. 1999), rather than open-loop high-intensity intermittent exercise or the reactive agility and skill performance that characterize many skill and (or) team sports. Although the existing studies did not show enhanced time to fatigue or exercise performance in either normal or stressful environments, the subjects' ratings of perceived exertion were occasionally reduced during exercise tasks while consuming BCAA (Blomstrand et al. 1997; Cheuvront et al. 2004).

Our study design allowed us to determine whether the fatigue-induced decrements in motor skill performance had a CNS component related to serotonin concentration. It is clear that our fatiguing protocol impaired handball performance, similar to the skill decrements in other team sports like soccer (Rampinini et al. 2008, 2009; Welsh et al. 2002), even in the presence of carbohydrate supplementation. We were unable to identify a substantial difference in the efficacy of the protein supplements because both depleting and PC supplements appeared to attenuate fatigue-induced decline in accuracy during the handball task (Fig. 2F). Reactive tasks are often performed by trading speed for accuracy, which can be accounted for by using the TPI. This index detected differences in performance because the PC and BL TPIs decline to a similar extent $(13 \%-16 \%)$ in response to fatiguing exercise, compared with $\sim 6 \%$ seen in the depleting trial. These data suggest that motor skill performance is affected by changes in brain neurotransmitter concentrations, but not necessarily serotonin. Both experimental treatments, although designed to "deplete" or "maintain" the brain with serotonin, contained significant quantities of tyrosine and phenylalanine, which are precursors to the neurotransmitter dopamine (Nathan et al. 2004), potentially masking the effect of serotonin depletion on postexercise skill performance. Further work is required to partition the effects of the different neurotransmitters and neurotransmitter precursors on exercise skill performance.

The exercise tests (RAT and RMST) reflect only discrete aspects of athlete decision making, and these are likely reactive decisions-responses as opposed to complex decisions made during games. We ascertained whether fatigue-induced changes in higher brain centres including mood states and cognitive function were attenuated by pre-exercise LNAA supplementation. Our findings and those of others (Hornery et al. 2007; Rampinini et al. 2008, 2009; Royal et al. 2006; Welsh et al. 2002; Winnick et al. 2005) demonstrate that $\operatorname{cog}$ nitive function and mood state are improved or impaired after fatiguing exercise, respectively, and may be inversely related to free tryptophan concentrations and therefore serotonin (Matrenza et al. 2004). The large individual variability of responses in the 2 questionnaire-based tests, especially regarding mood states (Table 3), makes it difficult to transfer these 
findings to other athletic settings. However, the depleting trial caused a greater increase in total mood disturbance scores postexercise compared with the BL trial (Table 3), suggesting that players felt worse. The significance of this change in total mood state disturbance is difficult to interpret because players demonstrated similar increases in mood disturbance scores or bad feelings after the PC. However, these changes in mood were aligned with the exercise test performances. Specifically, in the depleting trial, performance was enhanced after fatiguing exercise, in contrast to a decline in performance in the BL and PC trials. Furthermore, our data indicate that cognitive function after fatiguing exercise in the depleting trial was moderately enhanced, as indicated by the greatest Stroop Color-Word Score (Table 3). Taken together, these data provide evidence that high-dose LNAA supplements that either "deplete" or "maintain" the brain serotonin improve cognitive abilities but tend to negatively affect mood states in athletes after exercise.

It was important to determine the effects on plasma LNAA concentrations of the depleting and PC supplements used in this study because we were unable to quantify changes in brain serotonin levels in our athletes. In addition, our supplements differed from other studies in exercise (Blomstrand et al. 1991, 1997; Watson et al. 2004; Weicker and Struder 2001) and psychopharmacology (Harrison et al. 2004; Matrenza et al. 2004; Nathan et al. 2004). Moreover, quantifying changes in the free tryptophan/LNAA ratios provides the best indication of the level of serotonin depletion (Nathan et al. 2004; Pardridge 1998). Because of the differences in the timing of blood sampling, supplement ingestion, and intervention, it was difficult to make accurate comparisons among studies. However, when our plasma LNAA concentrations and free tryptophan/LNAA ratios were compared with other exercise studies, our postexercise amino acid concentrations and free tryptophan/LNAA ratios were equivalent to the reported data (Blomstrand et al. 1991; Watson et al. 2004). Furthermore, when comparing our resting data $(3 \mathrm{~h}$ postsupplement) with the cognitive function studies conducted at rest, again our supplements resulted in similar alterations in plasma LNAA concentrations for both the depletion and PC trials (Nathan et al. 2004; Young et al. 1985). It is therefore likely that our supplementation strategies influenced the uptake of tryptophan and potentially altered serotonin production-synthesis in the brain.

Although we have demonstrated an ergogenic effect of a tryptophan-depleting LNAA supplementation regimen, these results need to be considered in the context of the study limitations. The serotonin-depleting supplement used required players to ingest a very powdery suspension, making palatability difficult. This large quantity ( $\sim 44 \mathrm{~g})$ of amino acids would also be financially prohibitive to all but the best-sponsored elite athletes. Finally, given the mood-altering potential of these LNAA mixtures, it is recommended that athletes with diagnosed mood disorders like depression and bipolar disorder not undertake this supplementation strategy.

\section{Conclusion}

It appears that LNAA may have central effects on sportsspecific motor skills, cognitive function, and decision making. Specifically, the serotonin-depleting amino acid mixture improved some aspects of reactive motor skills performance and cognitive function when players were fatigued, compared with the no-supplement and the PC trial. Overall time to complete the RMST for the depleting intervention protein was $5 \%$ faster than BL, after fatiguing exercise. The depleting mixture lowered the free tryptophan/LNAA ratio after ingestion. Taken together, these results provide evidence of the benefits of high-dose amino acid supplementation on performance when ARF players are fatigued. This ergogenic effect could be especially useful during conditions of fatigue experienced at the end of quarters, halves, and games in team sport players. However, this supplementation regimen must be balanced with potential limitations, especially in individuals with possible mood disorders. Further research is required to elucidate the effects of tryptophan-serotonindepleting supplements of various doses on sports-specific decision making-cognitive function across a range of sports and athletic groups.

\section{Acknowledgements}

We thank the participants who gave up their valuable time for the study. Our gratitude is also extended to Natasha Gregory and Andrew Robinson from Monash University; Sam Schluter-Hughes, Cameron Tonkin, Ben Perry, Vicki Wyckelsma, Vanessa Leonie, Ian Fairweather, Dr. Rob Aughey, and Dr. Francois Billaut from Victoria University; Denise Thomas and Christopher Warren from Macquarie University; and Prof. Will Hopkins from Auckland University of Technology for their assistance with the large number of technical aspects in this study. The study was funded by the Australian Institute of Sport General and Collaborative Research Program. Amino acid powders, except where stated, were donated by Musashi (Nestle Australia).

\section{References}

Allen, D.G., Lamb, G.D., and Westerblad, H. 2008. Skeletal muscle fatigue: cellular mechanisms. Physiol. Rev. 88(1): 287-332. doi:10.1152/physrev.00015.2007. PMID:18195089.

Batterham, A.M., and Hopkins, W.G. 2006. Making meaningful inferences about magnitudes. Int. J. Sports Physiol. Perform. 1(1): 50-57. PMID:19114737.

Blomstrand, E., Celsing, F., and Newsholme, E.A. 1988. Changes in plasma concentrations of aromatic and branched-chain amino acids during sustained exercise in man and their possible role in fatigue. Acta Physiol. Scand. 133(1): 115-121. doi:10.1111/j. 1748-1716.1988.tb08388.x. PMID:3227900.

Blomstrand, E., Perrett, D., Parry-Billings, M., and Newsholme, E.A. 1989. Effect of sustained exercise on plasma amino acid concentrations and on 5-hydroxytryptamine metabolism in six different brain regions in the rat. Acta Physiol. Scand. 136(3): 473-481. doi:10.1111/j.1748-1716.1989.tb08689.x. PMID: 2473602.

Blomstrand, E., Hassmén, P., and Newsholme, E.A. 1991. Effect of branched-chain amino acid supplementation on mental performance. Acta Physiol. Scand. 143(2): 225-226. doi:10.1111/j. 1748-1716.1991.tb09225.x. PMID:1962526.

Blomstrand, E., Hassmen, P., Ek, S., Ekblom, B., and Newsholme, E.A. 1997. Influence of ingesting a solution of branched-chain amino acids on perceived exertion during exercise. Acta Physiol. Scand. 159(1): 41-49. doi:10.1046/j.1365-201X.1997.547327000.x. PMID:9124069.

Burke, L.M., and Hawley, J.A. 1999. Carbohydrate and exercise. 
Curr. Opin. Clin. Nutr. Metab. Care, 2(6): 515-520. doi:10.1097/ 00075197-199911000-00015. PMID:10678682.

Cheuvront, S.N., Carter, R. , 3rd, Kolka, M.A., Lieberman, H.R., Kellogg, M.D., and Sawka, M.N. 2004. Branched-chain amino acid supplementation and human performance when hypohydrated in the heat. J. Appl. Physiol. 97(4): 1275-1282. doi:10.1152/ japplphysiol.00357.2004. PMID:15358751.

Davis, J.M., and Bailey, S.P. 1997. Possible mechanisms of central nervous system fatigue during exercise. Med. Sci. Sports Exerc. 29 (1): 45-57. PMID:9000155.

Davis, J.M., Bailey, S.P., Woods, J.A., Galiano, F.J., Hamilton, M.T., and Bartoli, W.P. 1992. Effects of carbohydrate feedings on plasma free tryptophan and branched-chain amino acids during prolonged cycling. Eur. J. Appl. Physiol. Occup. Physiol. 65(6): 513-519. doi:10.1007/BF00602357. PMID:1483439.

Davis, J.M., Welsh, R.S., de Volve, K.L., and Alderson, N.A. 1999. Effects of branched-chain amino acids and carbohydrate on fatigue during intermittent, high-intensity running. Int. J. Sports Med. 20 (5): 309-314. doi:10.1055/s-2007-971136. PMID:10452228.

Farrow, D., Young, W., and Bruce, L. 2005. The development of a test of reactive agility for netball: a new methodology. J. Sci. Med. Sport, 8(1): 52-60. doi:10.1016/S1440-2440(05)80024-6. PMID: 15887901.

Gandevia, S.C. 2001. Spinal and supraspinal factors in human muscle fatigue. Physiol. Rev. 81(4): 1725-1789. PMID:11581501.

Harrison, B.J., Olver, J.S., Norman, T.R., Burrows, G.D., Wesnes, K. A., and Nathan, P.J. 2004. Selective effects of acute serotonin and catecholamine depletion on memory in healthy women. J. Psychopharmacol. 18(1): 32-40. doi:10.1177/0269881104040225. PMID:15107182.

Hopkins, W.G. 2000. Reliability from consecutive pairs of trials (Excel spreadsheet). In A new view of statistics. sportsci.org: Internet Society for Sport Science, http://sportsci.org/resource/ stats/index.html. [Accessed 1 October 2010.]

Hornery, D.J., Farrow, D., Mujika, I., and Young, W. 2007. Fatigue in tennis: mechanisms of fatigue and effect on performance. Sports Med. 37(3): 199-212. doi:10.2165/00007256-200737030-00002. PMID:17326696.

Jakeman, P.M. 1998. Amino acid metabolism, branched-chain amino acid feeding and brain monoamine function. Proc. Nutr. Soc. 57 (1): 35-41. doi:10.1079/PNS19980007. PMID:9571706.

Matrenza, C., Hughes, J.M., Kemp, A.H., Wesnes, K.A., Harrison, B.J., and Nathan, P.J. 2004. Simultaneous depletion of serotonin and catecholamines impairs sustained attention in healthy female subjects without affecting learning and memory. J. Psychopharmacol. 18(1): 21-31. doi:10.1177/0269881104040215. PMID: 15107181.

Meeusen, R., Watson, P., Hasegawa, H., Roelands, B., and Piacentini, M.F. 2006. Central fatigue: the serotonin hypothesis and beyond. Sports Med. 36(10): 881-909. doi:10.2165/00007256-20063610000006. PMID:17004850.

Mohr, M., Krustrup, P., and Bangsbo, J. 2003. Match performance of high-standard soccer players with special reference to development of fatigue. J. Sports Sci. 21(7): 519-528. doi:10.1080/ 0264041031000071182. PMID:12848386.

Moore, P., Landolt, H.P., Seifritz, E., Clark, C., Bhatti, T., Kelsoe, J., et al. 2000. Clinical and physiological consequences of rapid tryptophan depletion. Neuropsychopharmacology 23(6): 601-622. doi:10.1016/S0893-133X(00)00161-5. PMID:11063917.

Nathan, P.J., Hughes, J.M., McInerney, B., and Harrison, B.J. 2004.
Simultaneous depletion of tryptophan, tyrosine and phenylalanine as an experimental method to probe brain monoamine function in humans. Int. J. Neuropsychopharmacol. 7(2): 171-176. doi:10. 1017/S1461145703004073. PMID:14962353.

Nishizawa, S., Benkelfat, C., Young, S.N., Leyton, M., Mzengeza, S., de Montigny, C., et al. 1997. Differences between males and females in rates of serotonin synthesis in human brain. Proc. Natl. Acad. Sci. U S A 94(10): 5308-5313. doi:10.1073/pnas.94.10. 5308. PMID:9144233.

Pardridge, W.M. 1998. Blood-brain barrier carrier-mediated transport and brain metabolism of amino acids. Neurochem. Res. 23(5): 635-644. doi:10.1023/A:1022482604276. PMID:9566601.

Rampinini, E., Coutts, A.J., Castagna, C., Sassi, R., and Impellizzeri, F.M. 2007. Variation in top level soccer match performance. Int. J. Sports Med. 28(12): 1018-1024. doi:10.1055/s-2007-965158. PMID:17497575.

Rampinini, E., Impellizzeri, F.M., Castagna, C., Azzalin, A., Ferrari Bravo, D., and Wisløff, U. 2008. Effect of match-related fatigue on short-passing ability in young soccer players. Med. Sci. Sports Exerc. 40(5): 934-942. doi:10.1249/MSS.0b013e3181666eb8. PMID:18408603.

Rampinini, E., Impellizzeri, F.M., Castagna, C., Coutts, A.J., and Wisloff, U. 2009. Technical performance during soccer matches of the Italian Serie A league: effect of fatigue and competitive level. J. Sci. Med. Sport, 12(1): 227-233. doi:10.1016/j.jsams.2007.10. 002. PMID:18083631.

Royal, K.A., Farrow, D., Mujika, I., Halson, S.L., Pyne, D., and Abernethy, B. 2006. The effects of fatigue on decision making and shooting skill performance in water polo players. J. Sports Sci. 24 (8): 807-815. doi:10.1080/02640410500188928. PMID: 16815774

Spencer, M., Lawrence, S., Rechichi, C., Bishop, D., Dawson, B., and Goodman, C. 2004. Time-motion analysis of elite field hockey, with special reference to repeated-sprint activity. J. Sports Sci. 22 (9): 843-850. doi:10.1080/02640410410001716715. PMID: 15513278 .

Watson, P., Shirreffs, S.M., and Maughan, R.J. 2004. The effect of acute branched-chain amino acid supplementation on prolonged exercise capacity in a warm environment. Eur. J. Appl. Physiol. 93 (3): 306-314. doi:10.1007/s00421-004-1206-2. PMID:15349784.

Weicker, H., and Struder, H.K. 2001. Influence of exercise on serotonergic neuromodulation in the brain. Amino Acids, 20(1): 35-47. doi:10.1007/s007260170064. PMID:11310929.

Welsh, R.S., Davis, J.M., Burke, J.R., and Williams, H.G. 2002. Carbohydrates and physical/mental performance during intermittent exercise to fatigue. Med. Sci. Sports Exerc. 34(4): 723-731. doi:10.1097/00005768-200204000-00025. PMID:11932585.

Winnick, J.J., Davis, J.M., Welsh, R.S., Carmichael, M.D., Murphy, E.A., and Blackmon, J.A. 2005. Carbohydrate feedings during team sport exercise preserve physical and CNS function. Med. Sci. Sports Exerc. 37(2): 306-315. doi:10.1249/01.MSS.0000152803. 35130.A4. PMID:15692328.

Yamamoto, T., and Newsholme, E.A. 2000. Diminished central fatigue by inhibition of the L-system transporter for the uptake of tryptophan. Brain Res. Bull. 52(1): 35-38. doi:10.1016/S03619230(99)00276-2. PMID:10779700.

Young, S.N., Smith, S.E., Pihl, R.O., and Ervin, F.R. 1985. Tryptophan depletion causes a rapid lowering of mood in normal males. Psychopharmacology 87(2): 173-177. doi:10.1007/ BF00431803. PMID:3931142. 